\title{
The Optimal MAC Layer for Low-Power UWB is Non-Coordinated
}

\author{
Ruben Merz*, Alaeddine El Fawal*, Jean-Yves Le Boudec*, Božidar Radunović*, Jörg Widmer ${ }^{\dagger}$ \\ ${ }^{*}$ EPFL, School of Computer and Communication Sciences \\ \{ruben.merz, alaeddine.elfawal, jean-yves.leboudec, bozidar.radunovic\}@epfl.ch \\ $\dagger$ DoCoMo Euro-Labs \\ widmer@docomolab-euro.com
}

\begin{abstract}
We consider the design of the MAC layer for low power, low data-rate, impulse-radio ultra-wide band (IRUWB) networks. In such networks, the primary concern is energy consumption rather than rate efficiency. We explore several dimensions such as power control, rate adaptation, mutual exclusion, slotted versus non-slotted operation, power saving modes and interference mitigation. We analyze the effect of these design choices on the energy consumption and rate efficiency. We use a method of energy quanta for computing the energy consumption. We find that for both cases, the optimal operation is non-coordinated and with no power control. Sources should send at their maximum power and not pay attention to neighboring nodes. However, sources should constantly adapt their transmission rate to the level of interference.
\end{abstract}

\section{INTRODUCTION}

Emerging pervasive networks assume the deployment of large numbers of wireless nodes, embedded in everyday life objects. In these types of networks, the primary focus is on minimizing the energy consumption rather than maximizing rate.

There is a large design space for the medium access control (MAC) layer for low-rate, low-power UWB networks. Indeed the MAC may have access to some or all of the physical layer parameters. For instance, an important design choice is whether to allow interference (permit concurrent, interfering transmissions) or to enforce mutual exclusion. Another design choice is deciding whether to use power control. Also, how to coordinate nodes such that many of them can sleep? In the context of rate efficiency, [1] and [2] demonstrate that interference does not need to be completely prevented, but it needs to be managed (see Section II). The rate or the power can be dynamically adapted to the level of interference. In [2], rather than preventing interference, sources adapt their rate such that their destination can sustain the interference.

These choices have implications on the MAC layer, as well as the physical layer. Hence, there is a need to understand the design and implementation trade-offs. In this paper, we analyze the performance of various design choices from an energy consumption perspective.

The work presented in this paper was supported (in part) by the National Competence Center in Research on Mobile Information and Communication Systems (NCCR-MICS), a center supported by the Swiss National Science Foundation under grant number 5005-67322, and by CTI contract No7109.2;1 ESPP-ES.
We concentrate on large self-organized networks ${ }^{1}$. We focus on IR-UWB physical layer systems for low data-rate applications. These systems make use of ultra-short duration $(<1 \mathrm{~ns})$ pulses that yield ultra-wide bandwidth signals. They are characterized by a low duty cycle $(\simeq 1 \%)$ and extremely low power spectral densities [3]. Multi-user access is possible thanks to pseudo-random time hopping sequences (THS) that randomize the transmit time of each pulse. IR-UWB systems are especially attractive for low data-rate wireless communications as they potentially combine low power consumption, immunity to multipath fading and location/ranging capability

In Section II, we explore the design space of MAC protocols. In Section III we analyze the performance implications of fundamental design choices. We first propose a method for evaluating the energy consumption in the design phase of IRUWB systems (Sections III-A). We then derive a set of facts on the optimal design for low power UWB networks (Section III-C).

\section{The Design Space of The MAC LAYER}

The MAC layer globally manages the interference and medium access on a shared communication channel. Its main goal is to maximize in a fair manner both the overall lifetime of the network and the rate offered to each node. The MAC layer has to provide the three following functions:

- Interference Management: A source can control the interference it creates, or it can adapt to the existing level of interference.

- Access to a Destination: We assume that a node can receive from a single source at a time. Hence, an exclusion protocol becomes necessary to enforce that only one source communicates with the destination. This private exclusion protocol only involves the potential sources and the destination.

- Sleeping Management: This is the most effective way to conserve energy. There exists an important tradeoff between long sleep cycles (efficient energy savings) and short cycles (facilitate communication and improve responsiveness).

The large number of dimensions in the design of the MAC layer comes from the numerous possibilities available

\footnotetext{
${ }^{1}$ We do not address the case of Wireless Personal Area Networks (WPAN)
} 
to implement the above functions. In the following we give a list of six building blocks to implement the above functions. Each of them can contribute to to one or several functions.

1) Rate Adaptation: Often, the transmission rate is adapted as a function of the channel condition (essentially the attenuation) between the source and the destination. However, the rate can also be adapted as a function of the interference created by other devices in the network.

Note that rate control involves no nodes other than the source-destination pair.

2) Power Control: The transmit power can be adjusted to keep the signal-to-interference-and-noise ratio (SINR) at the destination constant, or to minimize the amount of interference created on the neighbors.

Contrary to rate control, power control requires interaction with other devices in the network.

3) Mutual Exclusion: A mutual exclusion protocol prevents nodes from transmitting at the same time. Most traditional protocols (for instance 802.11) use mutual exclusion to manage interference.

4) Multi-Channel: In a multi-channel protocol, the transmission medium is separated into several orthogonal or quasiorthogonal transmission channels. With IR-UWB, we have quasi-orthogonal channels thanks to the randomly chosen THS. Perfectly orthogonal channels inherently solve the traditional hidden-node terminal problem present in single-channel protocols. Still, in the case of quasi-orthogonal channels, the issue of the near-far effect appears; as for CDMA systems, the interference created by a strong interferer can still be nonnegligible for a receiver on another channel.

5) Multi-user Reception: With a multi-user receiver the near-far effect can be effectively addressed (see for instance [4] and reference therein). Unfortunately, multi-user reception has a high complexity and synchronization with all the sources to be decoded is necessary. However it is possible to exploit the structure of IR-UWB signals and derive suboptimal but much simpler techniques, such as interference mitigation [2]; interference mitigation takes advantage of the impulsive nature of the interference. Signals received with a much larger power than average are canceled. Their loss is recovered by the error correcting code at the cost a small rate reduction. Note that interference mitigation does not require synchronization with concurrent transmitters.

6) Sleeping: Slotted versus Unslotted Mechanisms: We consider two types of sleeping protocols. The first one is time slotted and uses a periodic beacon. This beacon provides a coarse-level synchronization. After the beacon, potential senders announce transmission requests. Receivers can then sleep except for the periods when announced transmissions occur.

The second approach is unslotted: each receiver wakes up according to its own listening schedule. A transmitter that wants to communicate with a given receiver needs to learn the listening schedule of this receiver. If all nodes have the same sleeping scheme (but delayed in time), a transmitter simply has to send a long preamble, as long as the maximum sleeping time.

\section{Performance Analysis of the Different Design CHOICES}

In this section we evaluate the effect of the design choices on the energy consumption and rate efficiency. Our general setting is low-power, low-rate IR-UWB networks. Our results are obtained either by review of the literature, or by adhoc analysis and simulations. But first we define the energy consumption model and performance metrics used in the analysis. Then we present six facts on the optimal design of low-power IR-UWB networks.

\section{A. Energy Consumption Model}

Our goal is to define an energy model that is independent of a given hardware implementation. This is a serious challenge, but we can take advantage of the nature of IR-UWB to derive a generic model, which is flexible enough to account for a large set of options.

With IR-UWB, time is divided into frames of $N_{c}$ short duration chips. (where one pulse is transmitted per frame). We can define a chip-level model of energy consumption. During a chip, the physical layer can either transmit a pulse, receive a pulse, perform signal acquisition, be in an active-off state, or sleep. The active-off state occurs due to time-hopping. When a node is between two pulse transmissions or receptions, energy is consumed only to keep the circuit powered up, but no energy is used for transmitting or receiving pulses.

Hence, we model the energy consumption by considering the energy per chip for each state. An energy consumption model is described by the vector

$$
\vec{q}=\left[\begin{array}{lll}
q_{t x} & q_{r x} & q_{a o}
\end{array}\right]
$$

where $q_{t x}$ is the cost for transmitting a pulse, $q_{r x}$ for receiving a pulse and $q_{a o}$ for being in the active-off state. Since the same transceiver elements are used for signal acquisition and reception, the acquisition energy consumption is also equal to $q_{r x}$. The cost while sleeping is negligible. It is fairly difficult to give precise figures for $\vec{q}$. Fortunately only relative values are relevant to our performance evaluation. It is thus possible to limit our analysis to the small set of scenarios described in Table I.

Example 1 (Energy consumption to receive a packet): For a packet of 127 bytes (including a synchronization preamble of 20 bytes) using binary modulation we have

$$
8 \cdot(\underbrace{20 \cdot N_{c} \cdot q_{r x}}_{\text {Preamble acquisition }}+\underbrace{107 \cdot q_{r x}}_{\text {Pulses reception }}+\underbrace{107 \cdot\left(N_{c}-1\right) \cdot q_{a o}}_{\text {Active-off state }})
$$

where the factor eight appears since we consider bytes.

With this model, the energy consumed for each received or transmitted packet can be easily computed. The lifetime of a node is then the time necessary to consume all the energy contained in the battery of the node. 
TABLE I

ENERGY CONSUMPTION MODEL FOR THE PERFORMANCE ANALYSIS

\begin{tabular}{|c|c|c|}
\hline \multicolumn{3}{|c|}{ Energy consumption models $\vec{q}=\left[\begin{array}{lll}q_{t x} & q_{r x} & q_{a o}\end{array}\right]$} \\
\hline 1 & $\vec{q}=\left[\begin{array}{lll}1 & 1 & 1\end{array}\right]$ & Baseline model \\
\hline 2 & $\vec{q}=\left[\begin{array}{lll}1 & 5 & 1\end{array}\right]$ & Higher cost for reception \\
\hline 3 & $\vec{q}=\left[\begin{array}{lll}1 & 1 & 0.5\end{array}\right]$ & Lower cost for active-off \\
\hline 4 & $\vec{q}=\left[\begin{array}{lll}1 & 5 & 0.5\end{array}\right]$ & Higher cost for reception, lower cost for active-off \\
\hline
\end{tabular}

\section{B. Performance Metrics and Simulation Parameters of the Performance Analysis}

1) Performance Metrics: The performance metric for the energy consumption is the sum of logs of node lifetimes. For rate efficiency, we use the sum of logs of average link rates. We use log utility metrics since they achieve a good tradeoff between efficiency and fairness [5].

2) Physical Layer and Simulations Parameters: For the simulations, we use an IR-UWB physical layer with timehopping. As such, we have quasi-orthogonal channel. The frame length is $N_{c}=1000$ chips, the chip duration is $T_{c}=1 \mathrm{~ns}$ and the energy per pulse is $0.2818 \mathrm{~mW}$. Thanks to puncturing on the error correcting code, the transmission rates ranges from $100 \mathrm{kbit} / \mathrm{s}$ to $1 \mathrm{Mbit} / \mathrm{s}$. We use the $802.15 .4 \mathrm{a}$ channel model 1 [6]. Rake receivers with perfect channel estimation are used with or without interference mitigation depending on the simulation setting. All nodes have the same physical layer, receiver design and the same initial battery power. Topologies for the simulation are static but randomly chosen on a 40 by 40 meter square. Further details can be found in [7] for the sleeping protocol and [2] for the error correcting code.

\section{Conclusion from the Performance Analysis: Facts About the Optimal Design}

We conduct our performance analysis by analyzing existing literature and by performing extensive simulations when needed. More details and our simulation code can be found in [8]. This leads us to the following six facts about the optimal design for low-rate, low-power UWB networks.

1) Rate control is needed: If the transmission rate is fixed, it has to be low enough to be feasible for the worst channel conditions. This in turn imposes the same low rate in good channel conditions. If transmission rates are low, packet transmissions last longer, and more energy is consumed to keep circuits running. This is highly inefficient from a lifetime or rate viewpoint (see [2]). As such rate control is necessary.

2) Power adaptation is not needed: Different power adaptation strategies for low-power UWB networks have been discussed in [5]. It is shown that any feasible rate allocation and energy consumption (hence lifetime) can be achieved with the $0 / P_{\max }$ strategy; whenever a node transmits data, it is with the maximum allowed transmission power $P_{\max }$.

Intuitively, since the signal-to-interference-and-noise ratio with impulse radio is convex in interference, increasing the transmit power of a source has more effect on the received signal at the destination than on interference on other nodes. As such, it is beneficial for a node to transmit with maximum power. This ensures a high rate and data transmissions terminate quickly. In contrast, using a lower transmit power extends the transmission duration. This is detrimental to reducing power consumption.

3) A suboptimal and simple form of multi-user detection is beneficial: At the cost of a small rate reduction, it was demonstrated in [2] and [9] that interference mitigation greatly alleviates the effect of one or several near-far interferers. Furthermore, it does not increase the energy consumption since only the power of the signal of interest needs to be measured.

4) Mutual exclusion is not needed when interference mitigation is applied: In case of near-far scenarios, it might seem desirable to enforce some form of mutual exclusion. But with interference mitigation, a large part of the interference can be eliminated. Hence, we simulate the impact of mutual exclusion on rate and lifetime with interference mitigation.

We assume each active receiver has a mutual exclusion region of radius $r$ around it; during reception, no node inside the exclusion region is allowed to transmit.

For each value of $r$ we find all the subsets of nodes that (1) minimize the energy consumption metric while still ensuring a minimum rate requirement and (2) satisfy the exclusion region constraints. Results for the lifetime are presented on Figure 1 for the baseline energy model (model 1, results are similar with the other energy models). With large value of $r$, the lifetime of the node is only slightly increased. Indeed, when rate constraints are low, each node transmits only during a small fraction of time. This in turn reduces the energy consumed to keep the circuits running. Hence the total interference created is small and the energy consumed is minimized. Furthermore, interference mitigation handles most of the interference, and there is no need to implement an exclusion protocol.

For the efficiency, the procedure is the following. For each value of $r$, we find all the subsets of nodes and rate of these nodes that (1) maximize the rate metric and (2) satisfy the exclusion region constraints. Results for the average rate achieved for different $r$ are also presented on Figure 1. It turns out it is optimal to let all nodes transmit concurrently at all times (the maximum is reached for $r=0$ ). Without interference mitigation, the optimal exclusion region size is approximately 2 meters. Thanks to interference mitigation, no mutual exclusion is required. The rate reduction due to interference mitigation is traded for an increased spatial reuse due to the absence of mutual exclusion.

5) Slotted sleeping is better than unslotted if occasional bursts must be supported: We consider the slotted and the unslotted sleeping protocol described in [7]. We analyze which protocol is more efficient in terms of average node lifetime. Details of the computations are reported in [7].

We compute the lifetime assuming that most of the time the node is subject to a load $\lambda_{0}$. However, the parameters are chosen to occasionally sustain a bursty traffic load $\lambda_{\max }>\lambda_{0}$ per receiver during burst intervals.

The slotted protocol performance depends on the the number $S_{A}$ of reservation slots [7]. The reservation slots are used by sources to announce their intention to transmit and to 


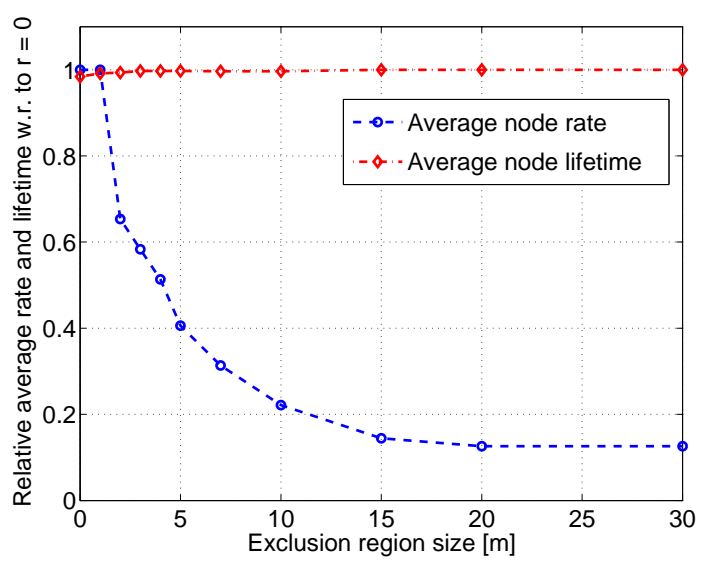

Fig. 1. Average node rate (dashed curve) and average node lifetime (dot dashed curve) relative to the values at $r=0$ versus the size of the exclusion region $r$. We use the energy model 1 . (Results are similar with the other energy models.) No exclusion region is required from a rate point of view. The presence of an exclusion region has a negligible impact on the lifetime of the node.

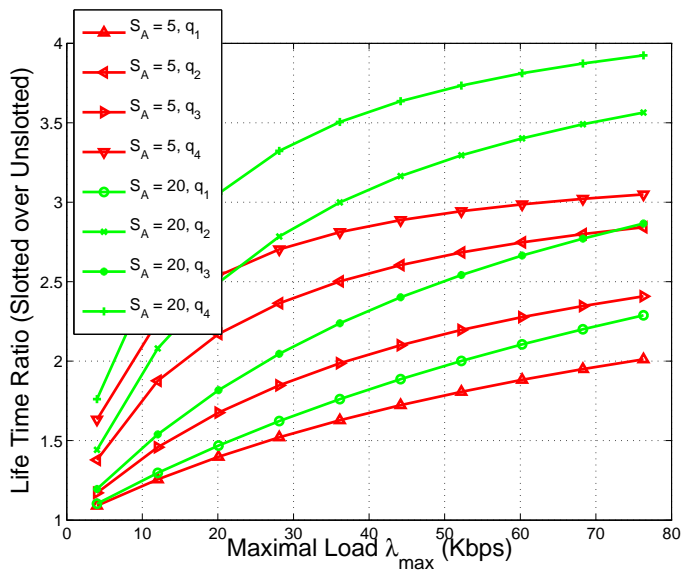

Fig. 2. Ratio of the average node lifetime in the slotted case over the unslotted case with respect to the maximal load $\lambda_{\max }$. We compare the performance for $S_{A}$ equal to 5 and 20 and all energy models (Table I). In all cases, the slotted protocol outperforms the unslotted one by $15 \%-30 \%$.

reserve subsequent transmission slots. Hence, for two extreme values of $S_{A}$ and the four energy models, we compare the lifetimes achieved with slotted and unslotted protocols with $\lambda_{0}=10 \mathrm{kbit} / \mathrm{s}$ (low duty cycle scenario). The ratios of the lifetime in the slotted over the unslotted case are plotted on Figure 2. With slotted sleeping protocols, the lifetime is $15 \%$ $50 \%$ longer. If the slotted structure comes at a low cost, or for free (as in a master-slave system like bluetooth), its use is optimal. If this is not the case, we need to compare the implementation overheads to compare the two protocols.

6) Unslotted sleeping is better than slotted if occasional maximum latency must be supported: We consider a variant of the previous section. We still assume that most of the time, the network is subject to an average traffic load $\lambda_{0}$. However, it has to occasionally support a small number of unpredicted, but very urgent messages instead of a bursty high load.

The ratios of the lifetime in the slotted case over the

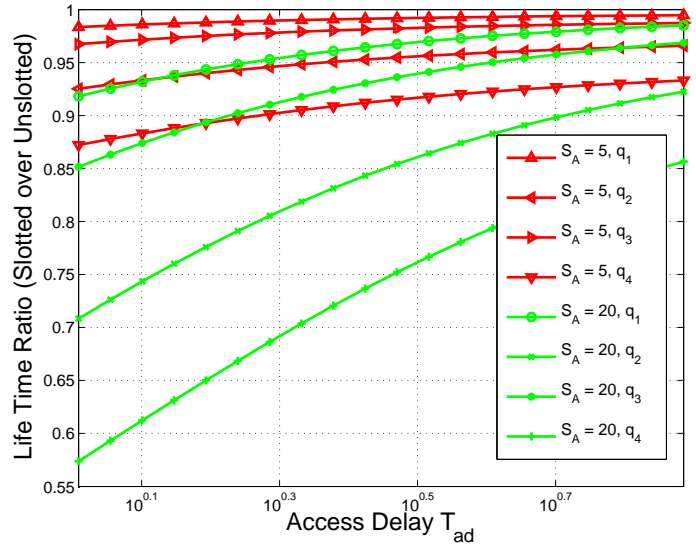

Fig. 3. Ratio of the average node lifetime in the slotted case over the unslotted case with respect to the access delay $T_{a d}$. We compare the performance for $S_{A}$ equal to 5 and 20 and all energy models (Table I). In this case, the unslotted protocol outperforms the slotted one.

unslotted case are plotted on Figure 3. The conclusions are the opposite of the previous section: the unslotted protocol always performs better or equal to the slotted protocol.

\section{CONCLUSiOnS}

In this paper, we analyzed the performance in terms of energy consumption of several design choices of the MAC layer. We developed a new energy consumption model for impulse radio systems

Our performance analysis lead us to six facts for the optimal design of low-rate, low-power IR-UWB networks. It clearly calls for an uncoordinated and decentralized protocol using rate adaptation and no power control.

\section{REFERENCES}

[1] F. Cuomo, C. Martello, A. Baiocchi, and F. Capriotti. Radio resource sharing for ad hoc networking with UWB. IEEE Journal on Selected Areas in Communications, 20(9):1722-1732, December 2002.

[2] R. Merz, J. Widmer, J.-Y. Le Boudec, and B. Radunovic. A joint PHY/MAC architecture for low-radiated power TH-UWB wireless adhoc networks. Wireless Communications and Mobile Computing Journal, Special Issue on Ultrawideband (UWB) Communications, 5(5):567-580, August 2005.

[3] M. Z. Win and R. A. Scholtz. Ultra-wide bandwidth time-hopping spreadspectrum impulse radio for wireless multiple-access communications. IEEE Transactions on Communications, 48(4):679-691, April 2000.

[4] Lang Tong, V. Naware, and P. Venkitasubramaniam. Signal processing in random access. IEEE Signal Processing Magazine, 21(5):29-39, September 2004.

[5] B. Radunovic and J. Y. Le Boudec. Optimal power control, scheduling and routing in UWB networks. IEEE Journal on Selected Areas in Communications, 22(7):1252-1270, September 2004.

[6] A.-F. Molisch, K. Balakrishnan, C.-C. Chong, S. Emami, A. Fort, J. Karedal, J. Kunisch, H. Schantz, U. Schuster, and K. Siwiak. IEEE 802.15.4a channel model - final report. Document 05/662r1, November 2004.

[7] A. El Fawal, J.-Y. Le Boudec, R. Merz, B. Radunovic, J. Widmer, and G. M. Maggio. Tradeoff analysis of PHY-aware MAC in low-rate, lowpower UWB networks. IEEE Communications Magazine, 43(12):147155, December 2005.

[8] UWB research at EPFL-IC. http://icawww1.epfl.ch/uwb/, 2006.

[9] A. El Fawal and J.-Y. Le Boudec. A power independent detection method for UWB impulse radio networks. In IEEE International Conference on Ultra-Wideband (ICU), 2005. 